\title{
5\% Ibuprofen Iontophoresis Compared with Transcutaneous Electrical Nerve Stimulation in the Management of Knee Osteoarthritis: A Feasibility Study
}

\author{
Ajediran I. Bello*, Shika Kuwornu \\ Department of Physiotherapy, School of Biomedical and Allied Health Sciences, College of Health Sciences, \\ University of Ghana, Accra, Ghana \\ Email: ${ }^{*}$ badmus@chs.edu.gh
}

Received 10 August 2014; revised 15 September 2014; accepted 8 October 2014

Academic Editor: Leung Kim Hung, Department of Orthopaedics \& Traumatology, Faculty of Medicine, the Chinese University of Hong Kong, China

Copyright @ 2014 by authors and Scientific Research Publishing Inc.

This work is licensed under the Creative Commons Attribution International License (CC BY).

http://creativecommons.org/licenses/by/4.0/

(c) ()

\section{Abstract}

Objective: The study compared the inclusions of 5\% ibuprofen iontophoresis and Transcutaneous Electrical Nerve stimulation (TENS) in the management of osteoarthritis (OA) of the knee joint. Subjects and Methods: Patients diagnosed with knee $O A$ and referred for physiotherapy at a tertiary health facility in Accra, Ghana participated in the study. They were alternately assigned into either TENS or iontophoresis groups. Patients in TENS Group received conventional TENS mode while those in iontophoresis group were treated with $5 \%$ ibuprofen iontophoresis using galvanic electrical current. Both protocols were performed twice weekly for six weeks in addition to their prescribed therapeutic exercises. 15-minute walking time, Numerical Rating Scale and Goniometer were used as the main outcome measures to determine subjects' walking speed, pain and active range of motion (AROM) respectively. Treatment evaluation was performed at baseline, week 3 and week 6 by an independent assessor. Comparisons of variables within and between groups were tested using Friedman's mean rank and Mann-Whitney $U$ tests respectively at $p<0.05$. Results: Participants in both groups were not significantly different in age $(p=$ $0.460)$, height $(p=0.548)$ and weight $(p=0.810)$. Significant improvements were recorded for walking speed $(p=0.001)$, pain $(p=0.001)$ and AROM $(p=0.001)$ of the

\footnotetext{
${ }^{*}$ Corresponding author.
}

How to cite this paper: Bello, A.I. and Kuwornu, S. (2014) 5\% Ibuprofen Iontophoresis Compared with Transcutaneous Electrical Nerve Stimulation in the Management of Knee Osteoarthritis: A Feasibility Study. Open Journal of Therapy and Rehabilitation, 2, 166-172. http://dx.doi.org/10.4236/ojtr.2014.24022 
subjects within groups. However, between group comparison of the treatment variables showed no significant differences $(p>0.05)$ for all the outcome measures. Conclusion: $5 \%$ ibuprofen iontophoresis and TENS add equal therapeutic value in the management of knee $0 A$. Consideration of both modalities as adjuncts to therapeutic exercises is therefore worthwhile for managing the condition.

\section{Keywords}

Ibuprofen, TENS, Osteoarthritis, Iontophoresis

\section{Introduction}

Osteoarthritis (OA) is a slowly progressive musculoskeletal disorder characterized by pain, joint stiffness, swelling, muscle weakness and joint instability thereby compromising mobility and active lifestyle [1]. Over $60 \%$ of the adult population in Ghana was reported to be affected by OA of the knee [2] thus presenting unique opportunity to improve its management strategies. The principal objectives of managing OA are to alleviate pain adequately, improve function and reduce disability [3]. Most clinical deficits associated with OA are amenable to conservative managements, including physiotherapy. Long-term application of these modalities is often performed either as a complimentary care to medical treatment or as a follow-up treatment to surgical intervention. Although, the use of supervised therapeutic exercises remains the most effective non-pharmacology modality for managing OA [4], existing data have lent credence to varying degree of efficacies of other modalities in physiotherapy [5]-[9]. Most often, transcutaneous electrical nerve stimulation (TENS), ultrasound, short-wave diathermy, iontophoresis, microwave diathermy, infra red rays among others are chosen as adjuncts to therapeutic exercise. However, selection of these options is somewhat based on personal preference.

TENS is commonly chosen by physiotherapists in Ghana in combination with exercises in the management of patients with knee OA. The therapeutic efficacy of TENS has been divergently reported in various clinical trials [7] [10] [11]. For instance, greater therapeutic gain of TENS is reported in the modulation of movement-evoked pain rather than pain at rest [5]. In addition to non-pharmacological management of OA, non-steroidal anti-inflammatory drugs are commonly prescribed by physicians to block the actions of prostaglandin-generating enzyme (Cyclooxygenase) which is the cardinal cause of inflammation and pain in OA [12]. Considering the prolonged oral intake of these drugs and its attendant adverse effects, iontophoresis is chosen as an alternative route through which patients with OA could benefit from such medications thereby preventing the development of unnecessary co-morbid conditions. It is a non-invasive procedure by which high concentration of ionisable substances, mostly medications or bio-active agents, is propelled transdermally by repulsive electromotive force generated from two electrodes fastened over the overlying skin of the body segment [13]. Existing data have shown therapeutic gains of this procedure when combined with therapeutic exercises [8] [9]. In spite of the documented therapeutic effects of TENS and iontophoresis however, their comparative efficacies in combination with exercise, have been minimally evaluated.

The global attention towards the rising trends of non-communicable disease coupled with increasing incidence of OA in Ghana, has necessitated the need to document the comparative effectiveness of the most readily available adjuncts modalities in the environment. This is envisaged to inform the physiotherapists of the appropriate selection of complimentary therapy to exercise in clinical practice. We therefore hypothesized that 5\% ibuprofen iontophoresis would produce a better treatment outcome than TENS application as an adjunct in the management of the knee OA.

\section{Materials and Methods}

\subsection{Study Design}

This is a feasibility intervention study design spanning a period of six weeks in which thirteen (13) patients were alternately assigned to either TENS $(n=7)$ or iontophoresis $(n=6)$ groups. 


\subsection{Subjects}

Patients diagnosed by resident physicians to have knee OA based on clinical and radiographic classification of the American College of Rheumatology, were invited to take part in the study at physiotherapy department, Korle-Bu Teaching Hospital, Accra. They were recruited through sample of convenience. To be included in the study, patients must present with activity-related pain on a Numerical Rating Scale (NRS) score of $\geq 4$ and have just been referred for physiotherapy. Patients with recent intra-articular injection, knee dysfunction secondary to systemic disease, pregnancy, metallic implants as well as open wound around the knee, were not considered for inclusion in the study. Of the 13 subjects invited, only ten completed the study: TENS group = 6; Iontophoresis $=4$. The remaining three dropped out on account of incompatibility with treatment protocols, ill-health or knee surgery.

\subsubsection{Instrument for Data Collection}

Multi-purpose electrical device (Enraf Nonius endomed 581 ID) was used to generate conventional TENS current and galvanic (direct) current for pain modulation and iontophoresis respectively. $200 \mathrm{ml}$ graduated medicine bottle (KANE-EM, Ghana) quantified $50 \mathrm{ml}$ of $5 \%$ ibuprofen solution for each participant during iontophoresis procedure. Active range of motion (AROM) of the affected knee of the participants was determined with halfcircle arm universal goniometer. 15-meter distance walkway marked on the floor served as the space for assessing the walking speed of the participants and stop watch (Xinjia, xj-621D) was used to determine the duration of the 15-meter walk time (15-mwt). Numerical Rating Scale (a straight line of $10 \mathrm{~cm}$ long) on a sheet of paper was adopted to assess the subjects' pain intensity.

\subsubsection{Procedure}

The protocol for this study was scrutinized and approved by the Ethics and Protocol Review Committees of the School of Allied Health Sciences, University of Ghana. Written informed consent was requested and obtained from the patients before taking part in the study. On the treatment days, each patient underwent physical examinations in supine lying position which include localization of painful spot through palpation, sensation test with an office pin and cotton wool to ascertain the sensitivity of the skin around the knee joint. To identify referred pain, participants were subjected to lower quarter screening using joint mobility and neurological tests. This procedure was also adopted to identify possible associated dysfunctions from other body segments capable of contributing to or perpetuating the symptoms at the knee.

\subsubsection{Baseline Assessment}

The baseline scores of the participants for active range of motion (AROM), walking speed and pain intensity were taken. The AROM was measured with patients in side lying and fully supported positions to ensure relaxation. The axis of the goniometer was placed at the lateral femoral epicondyle, while the stationary and mobile arms were positioned parallel to the thigh and the shank, respectively. The thigh girth of the affected lower limb was measured with participants in supine lying position and the affected lower limb positioned such that the patella faced the ceiling. The reference point for measurement was $15 \mathrm{~cm}$ above the base of patella. The 15-meter walking time was performed in a flat walkway corridor (15 m long and 3.4 wide) where both ends were properly marked with blue adhesive tape. Patients were asked to walk at their natural walking speed and to report any untoward feelings that could prevent them from completing the distance at their pace. Command was given by the investigators from the starting point to the end point while monitoring the duration of the walk through the stop watch. The patients were also asked to rate their pain as normally perceived during the activity using NRS.

\subsubsection{Exercise Therapy}

Therapeutic exercises were performed in the gymnasium at the physiotherapy department of the Hospital. The exercise modes included open and close kinetic chain training. Open kinetic chain exercises were performed in sitting and lying positions, and these included AROM exercises, quadricep drills (using quadriceps chair), cycling in air, alternate leg raise with knees at $90^{\circ}$, and straight leg raising, whereas close kinetic chain exercises included heel lifting, leg press exercises, wall-slide exercise, cycling, calf stretching, and gait training. Exercises were individually prescribed, and the principles of progression and overload were observed. Both groups had 30 minutes of active exercises twice weekly for six weeks. 


\subsubsection{Patient Preparation for TENS Application and Iontophoresis}

Patients in both groups were briefed about the protocol involved in the treatment viz.; sensation, duration and the effects to be perceived by them. The skin overlying the posterolateral and mediolateral aspects of the knee was cleaned with a wet warm towel to reduce the skin's resistance to electric current before the commencement of treatment. The area was further cleaned with methylated spirit to render it aseptic.

\subsubsection{TENS Application}

Patients were requested to assume supine lying position on the plinth. Two electrodes were placed lateromedially on either side of the knee and were secured in place with a Velcro strap covering the electrodes to ensure even distribution of pressure, and hence the current. Conventional TENS application (Phase duration $=200 \mu$ s; Frequency $=85 \mathrm{~Hz}$; Intensity $=10 \mathrm{~mA}$ ) was selected from the endomed 581 chronic preset programmes with treatment duration lasting 30 minutes per session [14]. Each participant was treated twice weekly for six weeks.

\subsubsection{Iontophoresis Process}

Patients in this group were treated with 5\% ibuprofen iontophoresis. They were well briefed about possible skin irritation arising from skin charge accumulation. All the participants agreed to take part based on the expected benefits from the procedure. Ibuprofen solution was prepared at pharmaceutical manufacturing unit of KBTH through the assistance of pharmacists. The negative electrode was saturated with $50 \mathrm{ml}$ of $5 \%$ ibuprofen solution while the positive electrode was moistened with water. Patients assumed similar position as described in the TENS group and the electrodes were similarly arranged. Continuous galvanic current was selected from the endomed 581 as follows: current density $=0.48 \mathrm{~mA} / \mathrm{cm}^{2}$, frequency $=8000 \mathrm{~Hz}$, phase interval $=5 \mu \mathrm{s}$, phase duration $=125 \mu$ s, duty cycle $=95 \%$ and treatment duration $=30$ minutes [12] [13] [15]. Participants' tolerance level was however considered in setting the current intensities in both groups. No complication was reported afterwards.

All participants were advised to adhere to their medication as directed by their physicians. Reassessments of outcome measures were performed at week 3 and week 6 of the clinical trial by an independent assessor.

\subsection{Data Analysis}

Data were analyzed with the SPSS version 20.0. The mean and standard deviations of all variables were calculated to summarize data. Comparison of physical characteristics of the subjects and the treatment outcomes on NRS, AROM, and 15-mwt between the two groups were tested using Mann-Whitney U test whilst Friedman's mean rank test was used to find any significant difference in the treatment outcome within the two groups. Significance level was set at 0.05 .

\section{Results}

Ten patients with unilateral OA were able to participate and complete the 6-week treatment regimen. The physical characteristics of the participants in the two groups were similar for age $(p=0.460)$, height $(p=0.548)$ and weight $(p=0.810)$ thus precluding extraneous factors that could place one group at advantage over the other. Results are presented in Table 1. Significant improvements were recorded within each group for pain ( $\mathrm{p}<$ 0.001), AROM ( $\mathrm{p}<0.001$ ) and 15 mwt $(\mathrm{p}<0.001)$ following six-week treatment evaluation period (Table 2). However, comparison of the two groups on VAS, AROM and 15 mwt did not show any significant differences at week 3 and week 6 .

\section{Discussion}

Osteoarthritis (OA) is one of the most common non-communicable diseases globally owing to its close tie with dysfunctions emanating from the biochemical, biomechanical and ageing process in human body system. Both surgical and conservative managements are commonly adopted by physicians and other relevant allied health care professionals in the management of patients with OA. Scientific evidence has established therapeutic exercises, dietary measure and health promotion as crucial in alleviating OA associated impairments [16]. Physiotherapists often prescribe therapeutic exercises along with other heat and electro-physical modalities as a means of alleviating the clinical deficits of the condition. Thus, the main aim of this study was to compare the treatment outcomes of TENS and iontophoresis inclusions, as popular adjuncts to therapeutic exercises, in the manage- 
Table 1. Mann-Whitney U test comparing physical attributes between the two groups.

\begin{tabular}{cccc}
\hline Treatment & Age $(\mathrm{yrs})$ & Height $(\mathrm{m})$ & Weight $(\mathrm{kg})$ \\
\hline TENS group & $58.20 \pm 8.70$ & $1.63 \pm 0.05$ & $81.40 \pm 5.59$ \\
Iontophoresis group & $53.40 \pm 7.89$ & $1.63 \pm 0.05$ & $84.20 \pm 10.38$ \\
Between groups analysis (p-value) & 0.460 & 0.548 & 0.810 \\
\hline
\end{tabular}

Table 2. Mean scores of variables within and between groups on the three outcome measures.

\begin{tabular}{|c|c|c|c|c|}
\hline Outcome measures & Baseline & Week 3 & Week 6 & p-value ${ }^{\mathrm{a}}$ \\
\hline NRS & $7.00 \pm 1.00$ & $4.40 \pm 0.89$ & $2.00 \pm 0.71$ & \\
\hline TENS group & $7.20 \pm 1.30$ & $4.80 \pm 1.10$ & $2.40 \pm 1.14$ & $0.001^{*}$ \\
\hline Iontophoresis group p-value ${ }^{\mathrm{b}}$ & $1.000^{*}$ & $0.762^{*}$ & $0.762^{*}$ & $0.001^{*}$ \\
\hline \multicolumn{5}{|l|}{ Goniometry } \\
\hline TENS group & $101.50 \pm 2.78$ & $107.10 \pm 3.61$ & $111.10 \pm 2.92$ & \multirow{2}{*}{$\begin{array}{l}0.001^{*} \\
0.001^{*}\end{array}$} \\
\hline p-value ${ }^{\mathrm{b}}$ & $0.635^{*}$ & $0.508^{*}$ & $0.286^{*}$ & \\
\hline \multicolumn{5}{|l|}{$15 \mathrm{mwt}$} \\
\hline TENS group & $30.60 \pm 2.70$ & $27.00 \pm 3.16$ & $23.60 \pm 2.97$ & \multirow{3}{*}{$\begin{array}{l}0.001^{*} \\
0.001^{*}\end{array}$} \\
\hline Iontophoresis group & $30.40 \pm 1.14$ & $27.20 \pm 1.10$ & $23.80 \pm 1.30$ & \\
\hline p-value ${ }^{\mathrm{b}}$ & 0.825 & 0.571 & 0.754 & \\
\hline
\end{tabular}

Key: ${ }^{*}=$ Significant; ${ }^{\mathrm{a}}=$ Friedman's mean rank test; ${ }^{\mathrm{b}}=$ Mann-Whitney U test.

ment of activated-related pain, AROM and walking speed of patients with knee OA. Our main finding shows no significant difference in the treatment outcomes of the inclusions of the two modalities. We therefore reject the set hypothesis that iontophoresis would produce better treatment outcome as an adjunct than TENS application in the management of the knee OA.

The patients in both groups demonstrated significant improvement in the pain, AROM and walking speed after six weeks of treatment. This finding buttresses the outcome of the previous clinical trials in which significant benefits of both modalities, in addition to other conservative treatment methods were reported [7] [8] [11] [17]-[19]. The concepts of pain modulation with TENS is anchored on Melzack and Wall's gate control theory as well as Sjolund and Eriksson's endorphin release theory [19]. The former is premised on the assumption that stimulation of the thick myelinated nerve fibers causes a neural inhibition at the spinal level which blocks the transport of pain stimuli to the brain via the non-myelinated nerve fibers (High intensity, low frequency TENS). The endorphin release theory on the other hand, stipulates that central nervous system can be stimulated to release endogenous opiates in the body thereby causing pain suppression (high-frequency, low-intensity). The recorded improvements in TENS group may be partly attributed to these physiological effects.

The treatment responses emanated from patients with the use of 5\% Ibuprofen iontophoresis followed similar trend as found in the earlier studies [8] [17] [18]. Xiaoying et al., reported that iontophoresis facilitated local and systemic delivery of ionisable drugs such as ibuprofen and sodium diclofenac, compared with passive diffusion. The maximum plasma concentration of these drugs, depending on the density of the applied current could be achieved within 1 hour of iontophoresis. The study also revealed that the concentrations of sodium diclofenac in the skin, subcutaneous tissue and muscle beneath the drug application site (cathode) were significantly greater than plasma concentrations and the concentrations of drug in similar tissues at the untreated sites. Allen recommended that in view of the adverse effects of prolonged, orally administered non-steroidal anti-inflammatory drugs (NSAIDS) on the gastrointestinal system of patients particularly, elderly OA patients, iontophoresis is a much safer means of offering drug therapy [18]. Study has shown that the ample depth of penetration of therapeutic concentrations of drugs into joints through iontophoresis was $1.7 \mathrm{~cm}$ and provides much higher local concentrations compared with oral medication and injections [12].

Clearly, the present trial has not been able to establish information different from those presented in the pre- 
vious efforts; its execution is a potential wakeup call to relevant healthcare professionals who are practicing in less technologically developed environment. Even though iontophoresis, as a well practiced procedure, has been overstressed in other geographical locations, investigating the possible variation in trend from other environments could enrich scientific knowledge about the topic. The boundaries between different groups of health care professionals are constantly shifting and there has been a discernible move in the recent years towards the twin notion of skill mixing and team approach in health care delivery. As the roles of physicians and allied health professionals change the demarcation line between them become increasingly blurred. These developments have spurred health care professionals to keep pace with the dynamic global health care trend through scientific research and debate.

\section{Limitation}

Although the present design aptly conforms to feasibility intervention, it suffers some major limitations. The sample size is acutely small due in part to the difficulty encountered in ensuring consistent follow up on the part of the patients and partly because of difficulty in getting freshly referred patients at the time this study was being conducted. The study design also appears to be weak owing to lack of randomization process which may have implications for its internal and external validity. The findings should therefore be taken within these limitations.

\section{Conclusion}

In view of the non-randomized design of this study, it is suggested that the inclusion of either $5 \%$ ibubrofen iontophoresis or TENS to other conservative methods be considered in the management of impairments associated with knee OA. Future efforts should therefore be based on a more rigorous design with the inclusion of disability measures and on longer period of evaluation.

\section{References}

[1] Veenhof, C. (2007) The Effectiveness of Behavioural Graded Activity in Osteoarthritis. Dutch Journal of Physical Therapy, 117, 118-119.

[2] Owusu-Ansah, B., Wiredu, E.K. and Hamzat, T.K. (2007) Comparative Efficacy of Transcutaneous Electrical Nerve Stimulation and Hotpack in the Management of Knee Joint Osteoarthritis. Ghana Journal of Allied Health Sciences, 1, 24-29.

[3] Vogels, E.M.H.M., Hendriks, H.J.M., vaa Baar, M.E., Dekker, J., HopmanRock, M. and Oostendorp, R.A. (2001) Clinical Practice Guidelines for Physical Therapy in Patients with Osteoarthritis of the Hip or Knee. KNGF-Guidelines for Physical Therapy in Patients with Osteoarthritis of the Hip or Knee.

[4] Fransen, M., McConell, S. and Bell, M. (2003) Exercise for Osteoarthritis of the Hip or Knee. Cochrane Database Systematic Review, 3, Article ID: CD004286.

[5] Vance, C.G., Rakel, B.A., Blodgett, N.P., DeSantana, M.J., Amendola, A., Zimmerman, M.B., et al. (2012) Effectiveness of Transcutaneous Electrical Nerve Stimulation on Pain, Pain Sensitivity and Function in People with Knee Osteoarthritis: A Randomized Controlled Trial. Physical Therapy, 92, 898-910. http://dx.doi.org/10.2522/ptj.20110183

[6] Rutjes, A.W., Nüesch, E. and Sterchi, R. (2009) Transcutaneous Electrostimulation for Osteoarthritis of the Knee. Cochrane Database Systematic Review, 4, Article ID: CD002823.

[7] Bello, A.I., Crankson, S. and Adegoke, B.O.A. (2014) Comparative Treatment Outcomes of Pre and Post-Exercise Tens Application on Knee Osteoarthritis: A Preliminary Report. Rehabilitation Process and Outcome, 3, 1-5. http://dx.doi.org/10.4137/RPO.S13794

[8] Aiyejusunle, C.B., Kola-Korolo, T.A. and Ajiboye, O.A. (2007) Comparison of the Effects of TENS and Sodium Salicylate Iontophoresis in the Management of Osteoarthritis of the Knee. Nigerian Quarterly Journal of Hospital Medicine, 17, 30-34. http://dx.doi.org/10.4314/nqihm.v17i1.12539

[9] Kivitz, A., Fairfax, M., Sheldon, E.A., Jone, B.A., Gammaitoni, A.R. and Gould, E.M. (2008) Comparison of the Effectiveness and Tolerability of Lidocaine Patch 5\% versus Celecoxib for Osteoarthritis-Related Knee Pain: Post Hoc Analysis of a 12 Week, Prospective, Randomized, Active-Controlled, Open-Label, Parallel-Group Trial in Adults. Clinical Therapeutics, 30, 2366-2377. http://dx.doi.org/10.1016/j.clinthera.2008.12.015

[10] Paker, N., Kesiktas, N. and Soy, D. (2006) Comparison of Therapeutic Efficacy of TENS versus Intra-Articular Hyaluronic Acid Injection in Patients with Knee Osteoarthritis. Advances in Therapy, 23, 342-353. http://www.ncbi.nlm.nih.gov/pubmed/16751166. cited 08/07/08 
[11] Itoh, K., Hirota, S., Katsumi, Y., Ochi, H. and Kitakoji, H. (2008) A Pilot Study on Using Acupuncture and Transcutaneous Electrical Nerve Stimulation to Treat Knee Osteoarthritis. Chinese Medicine, 3, 2. http://www.ncbi.nlm.nih.gov/pubmed/18312661. cited 08/07/08 http://dx.doi.org/10.1186/1749-8546-3-2

[12] Costello, C.T. and Jeske, AH. (1995) Iontophoresis: Applications in Transdermal Medication Delivery. Physical Therapy, 75, 554-563.

[13] Sarzi-Puttini, P., Cimmino, M.A., Scarpa, R. and Caporali, R. (2005) Osteoarthritis: An Overview of the Disease and Its Treatment Strategies. Seminars in Arthritis and Rheumatism, 35, 1-10. http://dx.doi.org/10.1016/j.semarthrit.2005.01.013

[14] Cameron, M.H. (2003) Physical Agents in Rehabilitation from Research to Practice. Saunders. St. Louis, Missouri, 227-230

[15] Cummings, J. (1987) Iontophoresis in Clinical Electrotherapy (Nelson R.M.) Norwalk. Appleton \& Lange, East Norwalk, 231-241.

[16] Balint, G. and Szebenyi, B. (1997) 9 Non-Pharmacological Therapies in Osteoarthritis. Bailliere's Clinical Rheumatology, 11, 1757-1761.

[17] Xiaoying, H., Anigbogu, A., Singh, P. and Xiong, G. (2001) Pharmacokinetic and Local Disposition of $\left[{ }^{14} \mathrm{C}\right]$ Sodium Diclofenac Following Iontophoresis and Systemic Administration in Rabbits. Journal of Pharmaceutical Sciences, 90, 1269-1276. http://dx.doi.org/10.1002/jps.1079

[18] Allen, L.V. (2007) Compounding for Geriatric and Arthritis Patients. Secundum Artem, 9. http://www.paddocklabs.com

[19] Low, J. and Reed, A. (2004) Electrotherapy Explained: Principles and Practice. 2nd Edition, Butterworth-Heinemann, Oxford. 
Scientific Research Publishing (SCIRP) is one of the largest Open Access journal publishers. It is currently publishing more than 200 open access, online, peer-reviewed journals covering a wide range of academic disciplines. SCIRP serves the worldwide academic communities and contributes to the progress and application of science with its publication.

Other selected journals from SCIRP are listed as below. Submit your manuscript to us via either submit@scirp.org or Online Submission Portal.
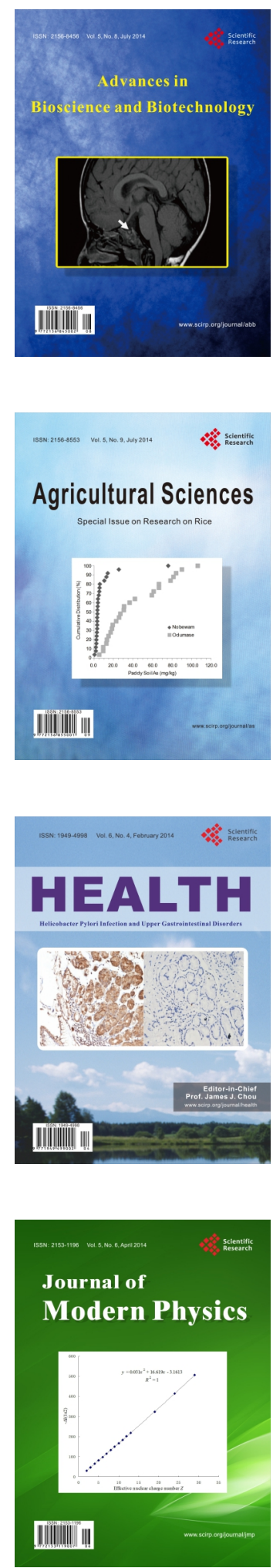
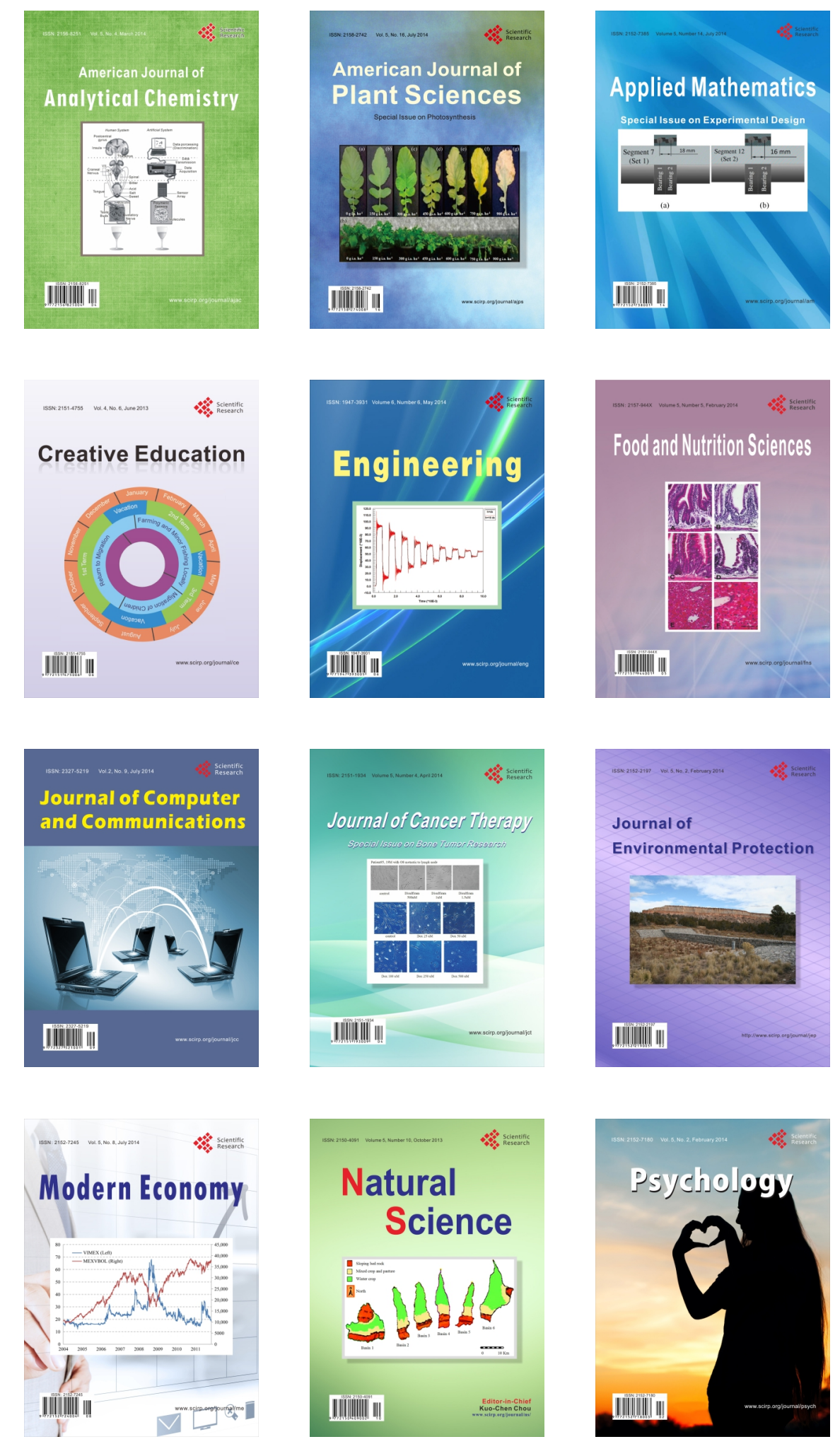